\title{
IMPACTO DA ORIENTAÇÃO FARMACÊUTICA NO PROCESSO \\ DE USO DE ANTICONVULSIVANTES POR CUIDADORES DE PACIENTES PEDIÁTRICOS COM EPILEPSIA REFRATÁRIA: ESTUDO DE VIABILIDADE
}

\author{
IMPACT OF PHARMACIST LED ANTICONVULSANT \\ COUNSELING FOR CAREGIVERS OF PEDIATRIC PATIENTS \\ WITH REFRACTORY EPILEPSY: A FEASIBILITY STUDY
}

\author{
Bruna Bergmann Santos ${ }^{1}$, Giovanna Webster Negretto ${ }^{1}$, \\ Lucas Miyake Okumura ${ }^{2,3}$
}

\section{Resumo}

Introdução: O estudo tem por objetivo avaliar o impacto da orientação do farmacêutico sobre o uso correto de anticonvulsivantes administrados por cuidadores de pacientes pediátricos com epilepsia refratária ao tratamento de primeira linha.

Métodos: Coorte prospectiva realizada em um hospital no sul do Brasil por 10 meses com todos os cuidadores principais dos pacientes $\leq 18$ anos internados na unidade de internação pediátrica, em uso domiciliar de 2 anticonvulsivantes orais ou mais, em que pelo menos um deles necessitava de adequação de forma farmacêutica. O processo de uso dos anticonvulsivantes foi avaliado através do instrumento Medtake Test, que pontua de 0 a $100 \%$ o conhecimento e a habilidade do cuidador no processo de uso dos medicamentos, no momento da internação, após a orientação farmacêutica de alta e por contato telefônico uma semana após a alta.

Resultados: 25 cuidadores de pacientes pediátricos foram incluídos e 22 completaram o estudo, em razão da perda de acompanhamento no período pós-alta dos pacientes. A média do escore Medtake foi de $77,5 \%( \pm 21,3 \%)$. Após a orientação farmacêutica, o escore médio aumentou cerca de $15 \%(p=0.02)$. Os principais tipos de erro identificados no estudo foram relacionados à administração dos medicamentos pela sonda, à utilização dos comprimidos sulcados partidos ao meio e à administração dos medicamentos em horários inadequados.

Conclusão: A magnitude do efeito positivo atribuível à orientação do farmacêutico foi de $15 \%$ sobre o processo de uso de medicamentos. Tal resultado é relevante para validação do Medtake Test no Brasil, além de cálculo amostral para estudos de intervenção sobre serviços farmacêuticos conduzidos no país para essa população.

Palavras chaves: Farmácia clínica; Pediatria; Epilepsia; Alta hospitalar

\section{Abstract}

Introduction: The study aims to evaluate the impact of pharmacist-led counseling on the correct use of anticonvulsants given by caregivers to pediatric patients with epilepsy refractory to first-line treatment.

Methods: A prospective cohort study was performed in a hospital in southern Brazil for 10 months with all the main caregivers of patients $\leq 18$ years hospitalized in the pediatric hospitalization unit, using 2 or more oral anticonvulsants at home and at least one of them needed pharmaceutical adjustment. The process of using anticonvulsants was evaluated through the Medtake test (which scores knowledge and ability of the caregiver in medication use process from 0 to 100\%) at the time of hospitalization, after pharmacist-led counseling, and 1 week after discharge by telephone contact.
Clin Biomed Res. 2021;41(2):107-116

1 Hospital de Clínicas de Porto Alegre. Porto Alegre, RS, Brasil.

2 Hospital Infantil Pequeno Príncipe. Curitiba, PR, Brasil.

3 MAPESolutions. São Paulo, SP, Brasil.

Autor correspondente: Bruna Bergmann Santos brunabergmannsantos@yahoo. com.br

Hospital de Clínicas de Porto Alegre Rua Ramiro Barcelos, 2350 90035-007, Porto Alegre, RS, Brasil. 
Santos et al.

Results: Twenty-five caregivers of pediatric patients were included and 22 completed the study because of loss to followup in the post-discharge period. The mean Medtake score was $77.5 \%( \pm 21.3 \%)$. After pharmacist-led counseling, the mean score increased by approximately $15 \%(p=0.02)$. The main types of errors identified in the study were related to drug administration by enteral tube, use of split scored tablets, and drug administration at inappropriate times.

Conclusion: The magnitude of the positive effect attributable to pharmacist-led counseling was $15 \%$ over the drug use process. This result is relevant for validation of the Medtake test in Brazil, as well as for sample calculation for intervention studies on pharmaceutical services conducted in the country for the pediatric population.

Keywords: Clinical pharmacy; Pediatrics; Epilepsy; Hospital discharge

\section{INTRODUÇÃO}

A epilepsia é caracterizada como uma doença crônica, com longo tempo de tratamento e, portanto, a adesão à farmacoterapia e o uso correto dos medicamentos estão fortemente relacionados ao controle adequado das convulsões, melhor qualidade de vida e menor risco de eventos adversos ${ }^{1,2}$. Embora um número cada vez maior de anticonvulsivantes venha surgindo no mercado para o tratamento da epilepsia infantil, a adaptação das formas farmacêuticas para esta população não ocorre na mesma extensão, dificultando o uso correto destes medicamentos ${ }^{3}$.

Muitos fatores podem estar relacionados a não adesão à terapia anticonvulsivante, tais como a não compreensão da prescrição médica, a utilização de regimes terapêuticos complexos, problemas socioeconômicos e até mesmo experiências negativas com tratamentos prévios. Cabe ressaltar que a adesão ao tratamento em pacientes pediátricos depende de seus cuidadores, sendo a educação em saúde parte indispensável para a promoção do uso correto de anticonvulsivantes e prevenção de agravos relacionados ${ }^{4}$.

Para a promoção do correto uso de medicamentos e diminuição de erros envolvendo medicamentos na transição do cuidado hospital-comunidade, ressaltamos a orientação realizada por farmacêuticos clínicos na alta hospitalar. Tal orientação pode auxiliar no esclarecimento de dúvidas dos cuidadores, facilitar a compreensão do receituário médico e com isso reduzir os erros envolvendo medicamentos após a alta, sendo uma ferramenta importante para aumentar a segurança do paciente em âmbito ambulatorial ${ }^{5-7}$. Embora esta seja uma prática rotineira em hospitais onde há farmacêuticos clínicos trabalhando em unidades pediátricas, artigos que envolvem educação farmacêutica na alta destes pacientes ainda são escassos, especialmente no Brasil. A literatura internacional já demonstrou a importância do farmacêutico na melhoria significativa do conhecimento sobre a doença e tratamento da epilepsia em cuidadores destes pacientes, resultando inclusive em maior confiança em administrar os medicamentos ${ }^{8}$.

Crescentemente os hospitais vêm investindo em programas de planejamento de altas multiprofissionais para pacientes pediátricos com condições clínicas complexas, onde diversas ferramentas e técnicas podem ser utilizadas para promover o uso correto de medicamentos, tais como o uso do método "teach back", confecção de calendários, materiais educativos impressos, seringas orais marcadas e adaptação de embalagens de medicamentos líquidos de fácil mensuração. ${ }^{9-13}$

Neste contexto o presente estudo de viabilidade visa avaliar o impacto da orientação do farmacêutico sobre o uso correto de anticonvulsivantes administrados por cuidadores de pacientes pediátricos com epilepsia refratária ao tratamento de $1^{\mathrm{a}}$ linha. Além disso, este estudo visa explorar a melhora no processo de uso de anticonvulsivantes dos cuidadores por meio da ferramenta Medtake Test, de forma a colaborar com cálculo amostral e futuros estudos conduzidos no Brasil ou com esse teste.

\section{Metodologia}

\section{Desenho do estudo e amostra}

Foi realizada uma coorte prospectiva com pacientes com $<18$ anos admitidos em uma unidade de internação pediátrica de um hospital universitário no período de dezembro de 2017 a setembro de 2018. Para inclusão no estudo, os pacientes deveriam estar em uso de farmacoterapia complexa para epilepsia refratária a $1^{\circ}$ linha de tratamento com uso domiciliar de dois anticonvulsivantes orais ou mais, onde pelo menos um deles necessite de adequação de forma farmacêutica. Tal critério se deve à maior chance de erros processuais no preparo e administração dos medicamentos, em especial situações onde há fracionamento de dose ou a necessidade de administrar medicamentos via sonda, gastrostomia ou jejunostomia. Não foram incluídos no estudo pacientes sem os cuidadores principais presentes na internação, pacientes com condições psiquiátricas ou que permaneceram por mais de duas semanas na Unidade de Terapia Intensiva ou que já haviam sido incluídos previamente no estudo sem que houvesse alteração nas prescrições dos anticonvulsivantes que necessitavam de adequação de forma farmacêutica. 


\section{Procedimento}

Todos os cuidadores principais dos pacientes incluídos no estudo foram avaliados por farmacêutico, o que incluiu uma entrevista (questionário pré-estruturado) para obtenção de informações sociodemográficas (idade, escolaridade, grau de parentesco com o paciente, número de filhos, se o cuidado com os medicamentos do paciente é compartilhado ou exclusivo e se já recebeu orientação farmacêutica prévia). Também foi realizada uma autoavaliação sobre a capacidade de administrar os medicamentos do paciente (nota de 0-10). No prontuário eletrônico foram obtidas informações clínicas (sexo, idade, diagnóstico) e da farmacoterapia (dose, posologia e tempo de uso dos medicamentos, via de administração, número total de medicamentos em uso domiciliar na admissão, número de medicamentos que necessitam de adequação de forma farmacêutica).

O instrumento Medtake Test foi utilizado para a avaliação da capacidade dos cuidadores principais dos pacientes em preparar e administrar adequadamente a farmacoterapia, em especial os anticonvulsivantes que necessitam de adequação de forma farmacêutica. Tal instrumento foi validado por Raehl et al. em $2002 \mathrm{e}$ foi adaptado para este estudo de forma a contemplar o preparo e administração dos medicamentos que necessitavam adequação de forma farmacêutica em pediatria ${ }^{14}$.

As adaptações no instrumento Medtake Test foram feitas na organização das etapas avaliadas relacionadas ao preparo e administração dos medicamentos, visto que para avaliar o uso corretos dos medicamentos pelos cuidadores, seria essencial avaliar cada etapa. Por exemplo, a forma de diluir o comprimido (considerando o tipo de liquido utilizado, a forma de "agitar" a suspensão obtida) e também no momento de administrar pela sonda (importante considerar a lavagem adequada da sonda e pausa na dieta enteral). Como a população escolhida para o estudo é muito especifica e há poucos estudos na literatura que avaliam a educação de cuidadores pediátricos quanto ao preparo e uso dos medicamentos de maneira geral, não encontramos material validado para a realização do estudo neste perfil de paciente. Foi utilizado um material adaptado de outra realidade, mas que com base nos resultados aqui apresentados será possível considerar o uso do material e validá-lo futuramente.

O teste consiste em uma descrição e demonstração detalhada do processo de utilização de cada medicamento, onde um farmacêutico treinado avalia o seu preparo, a posologia, a coadministração com água ou alimentos e se o cuidador responsável compreende a sua indicação. Para cada um destes elementos, conforme Raehl et al. ${ }^{14}$ em 2002, foi considerado um escore de $25 \%$ designado pelo farmacêutico avaliador, levando em conta as boas práticas de manipulação no preparo das diluições e a prescrição médica ${ }^{14,15}$. Ao final do teste foi atribuído um escore de $0-100 \%$ para cada medicamento. Além disso, a média de todos os medicamentos do paciente compõe o escore do Medtake Test.

A aplicação do instrumento foi realizada em três momentos: na internação ("baseline"), após a orientação farmacêutica de alta hospitalar ("alta") e uma semana após a alta hospitalar por telefone ("telefone"). No momento da alta também foi realizada uma autoavaliação dos cuidadores sobre a capacidade em administrar os medicamentos do paciente (nota de 0-10). Para a simulação do preparo das diluições na internação e antes da alta hospitalar foram fornecidos os materiais necessários. $\mathrm{Na}$ terceira etapa, realizada por contato telefônico, os cuidadores descreveram o preparo e administração dos anticonvulsivantes em casa e, quando o entrevistador julgasse necessário para melhorar a acurácia da avaliação, foram realizadas perguntas direcionadas. As etapas do estudo encontram-se resumidas na Figura 1. Cabe ressaltar que sempre que o avaliador julgasse necessário, foi realizado reforço de orientação e sanadas quaisquer dúvidas.
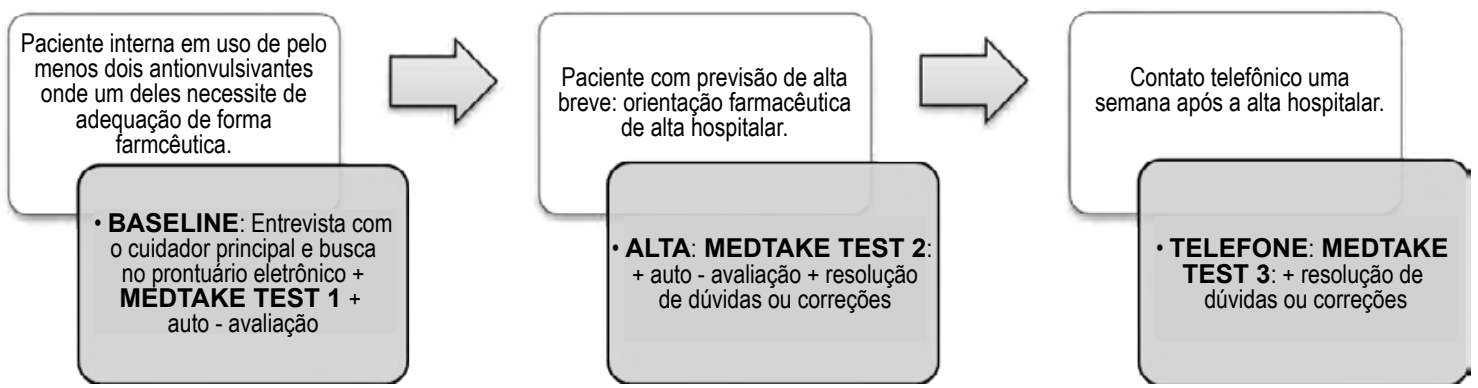

Figura 1: Passo a passo do estudo realizado e descrição dos três momentos principais do estudo.

*A orientação farmacêutica é personalizada e abrange a orientação "face a face" sobre o preparo dos medicamentos, a correta administração, os horários de uso e a forma de adquirir estes medicamentos após a alta hospitalar. Em todas as orientações, foi confeccionado pelo farmacêutico um material educativo impresso personalizado para cada paciente, contendo informações a respeito do preparo, administração, conservação e aquisição dos medicamentos. Além disso, foram disponibilizadas para os cuidadores seringas dosadoras orais previamente marcadas com as doses correspondentes a cada medicamento, marcadas por fitas de cores diferentes 
Este estudo foi aprovado pelo Comitê de Ética em Pesquisa do Hospital de Clínicas de Porto Alegre e o mesmo foi iniciado somente após a sua aprovação (CAAE: 52341416.0.0000.5327). Todo o cuidador principal foi convidado a participar do estudo e orientado quanto à pesquisa, sendo incluído no estudo somente após a assinatura do termo de consentimento livre e esclarecido.

\section{Análise de dados}

Todas as variáveis foram analisadas quanto a sua normalidade (teste de Kolmogorov-Smirnov) e reportadas por meio de estatística descritiva (médias, medianas, desvios padrões e intervalos interquartílicos).

Foi testada a hipótese alternativa de que a orientação do farmacêutico melhoraria o escore de Medtake test. Portanto, foi aplicado teste de KruskalWallis e Friedman para avaliar as médias do Medtake durante os três períodos avaliados (baseline, alta e telefone). Para fins de rejeição da hipótese nula foi considerado valor corte de $p<0,05$.

lém disso, foram realizadas correlações de Spearman (dados não paramétricos) para investigar a associação entre variáveis clínicas dos pacientes e escore de Medtake test. Nesta análise, valores de $p<0,05$ foram considerados correlações com significância estatística. A amostra utilizada foi de conveniência.

\section{Resultados}

\section{Características dos cuidadores e pacientes incluídos}

Ao todo, 25 cuidadores de pacientes pediátricos foram incluídos e 22 completaram o estudo por perda de acompanhamento dos pacientes no período pós- alta. Conforme a Tabela 1, a maioria $(92 \%)$ dos cuidadores eram mães dos pacientes, com idade média de $31,24(+9,14)$ anos, e 2 filhos, das quais $32 \%$ não completaram o ensino fundamental. Destes cuidadores, 17 (68\%) já haviam recebido em algum momento orientação farmacêutica em internações prévias e 14 (56\%) compartilhavam os cuidados do paciente com outros membros familiares.

Tabela 1: Características dos pacientes e cuidadores.

\begin{tabular}{lcc}
\hline & $\mathrm{N}$ & $\%$ \\
\hline Idade do paciente, anos, média (dp)* & 5,1 & 4,2 \\
Sexo, feminino & 13 & $52 \%$ \\
Diagnóstico neurológico & & \\
$\quad$ Epilepsia & 11 & $44 \%$ \\
Síndrome de West & 4 & $16 \%$ \\
Sem diagnóstico neurológico definido & 2 & $8 \%$ \\
\hline & \multicolumn{3}{c}{ Continua... }
\end{tabular}

Tabela 1: Continuação

\begin{tabular}{lcc}
\hline & $\mathrm{N}$ & $\%$ \\
\hline Esquizencefalia & 2 & $8 \%$ \\
Outras & 6 & $24 \%$ \\
Motivo admissão hospitalar & & \\
Pneumonia & 10 & $40 \%$ \\
Procedimento eletivo & 2 & $8 \%$ \\
Sepse & 2 & $8 \%$ \\
Mau controle das crises convulsivas & 2 & $8 \%$ \\
Esforço respiratório & 2 & $8 \%$ \\
Outros & 7 & $28 \%$
\end{tabular}

\section{Comorbidades**}

\begin{tabular}{|c|c|c|}
\hline Asma & 6 & $24 \%$ \\
\hline $\begin{array}{l}\text { Atraso do desenvolvimento } \\
\text { neuropsicomotor }\end{array}$ & 7 & $28 \%$ \\
\hline Distúrbio da deglutição & 3 & $12 \%$ \\
\hline Doença do refluxo Gastroesofágico & 3 & $12 \%$ \\
\hline Diabetes insipidus & 2 & $8 \%$ \\
\hline Faringo/Laringomalácea & 2 & $8 \%$ \\
\hline Distúrbio da deglutição & 2 & $8 \%$ \\
\hline Síndrome de Down & 2 & $8 \%$ \\
\hline Outros & 10 & $40 \%$ \\
\hline \multicolumn{3}{|l|}{ Parentesco cuidador } \\
\hline Mãe & 23 & $92 \%$ \\
\hline Pai & 2 & $8 \%$ \\
\hline dade do cuidador, anos, média (dp) & 31,24 & 9,14 \\
\hline Júmero de filhos, média (dp) & 2,4 & 1,4 \\
\hline \multicolumn{3}{|l|}{ Suidado } \\
\hline Exclusivo & 11 & $44 \%$ \\
\hline Compartilhado & 14 & $56 \%$ \\
\hline \multicolumn{3}{|l|}{ Escolaridade } \\
\hline Fundamental incompleto & 8 & $32 \%$ \\
\hline Fundamental completo & 4 & $16 \%$ \\
\hline $2^{\circ}$ grau incompleto & 5 & $20 \%$ \\
\hline $2^{\circ}$ grau completo & 7 & $28 \%$ \\
\hline Superior incompleto & 1 & $4 \%$ \\
\hline \multicolumn{3}{|l|}{ Drientação farmacêutica prévia } \\
\hline Sim & 17 & $68 \%$ \\
\hline Não & 8 & $32 \%$ \\
\hline
\end{tabular}

*dp: desvio padrão

**As comorbidades possuem contagens múltiplas para um mesmo paciente.

Em relação aos pacientes $(n=25)$, a maioria eram meninas $(52 \%)$ e com idade média de $5,1(+4,2)$ anos. Os diagnósticos de base mais comuns foram epilepsia refratária (44\%), seguido por Síndrome de West $(16 \%)$. As comorbidades mais frequentes foram atraso do desenvolvimento neuropsicomotor 
(28\%) e asma (24\%). De forma geral, os pacientes foram admitidos ao hospital por pneumonia (40\%).

\section{Perfil do uso de medicamentos na admissão e alta}

$\mathrm{Na}$ admissão, os pacientes estavam em uso de 5 [4-7] medicamentos na internação, sendo que, $50 \%$ necessitavam adequar a forma farmacêutica de 2 [2-3] medicamentos. Destes $44 \%$ usavam sonda e $40 \%$ usavam gastrostomia como via de administração dos medicamentos. $\mathrm{Na}$ alta, os pacientes tinham uma mediana de 5 [4-6] medicamentos prescritos, onde 2 [1-3] necessitavam de adequação de forma farmacêutica. No momento da alta metade dos pacientes utilizavam estes medicamentos pela sonda e metade pela gastrostomia.

No momento da internação foram avaliados pelo instrumento Medtake Test um total de 40 medicamentos anticonvulsivantes, sendo o clobazam o medicamento mais frequente $(42,5 \%)$, seguido pelo topiramato $(30 \%)$. Após a orientação farmacêutica foram avaliados 37 medicamentos, sendo o clobazam o medicamento mais encontrado $(45,9 \%)$. As características da farmacoterapia encontram-se detalhadas na Tabela 2. Apenas para um paciente foi incluído um novo anticonvulsivante e houve aumento de dose dos anticonvulsivantes avaliados durante a internação em 3 pacientes.

Tabela 2: Características da farmacoterapia avaliada.

\begin{tabular}{|c|c|c|}
\hline & $\mathrm{N}$ & $\%$ \\
\hline $\mathrm{n}^{\circ}$ Total medicamentos na internação, mediana (IQR*) & 5 & [4-7] \\
\hline $\begin{array}{l}n^{\circ} \text { Total medicamentos que necessitavam de adequação de forma farmacêutica na internação, } \\
\text { mediana }\left(\operatorname{IQR}^{\star}\right)\end{array}$ & 2 & [2-3] \\
\hline \multicolumn{3}{|l|}{$\%$ Medicamentos avaliados na internação } \\
\hline Clobazam & 17 & $42,5 \%$ \\
\hline Topiramato & 12 & $30,0 \%$ \\
\hline Vigabatrina & 5 & $12,5 \%$ \\
\hline Primidona & 2 & $5,0 \%$ \\
\hline Levetiracetam & 1 & $2,5 \%$ \\
\hline Lamotrigina & 2 & $5,0 \%$ \\
\hline Fenitoina & 1 & $2,5 \%$ \\
\hline Total de medicamentos avaliados & 40 & $100,0 \%$ \\
\hline $\mathrm{n}^{\circ}$ Total medicamentos na alta, mediana $\left(I Q R^{*}\right)$ & 5 & {$[4-6,25]$} \\
\hline $\begin{array}{l}n^{\circ} \text { Total medicamentos que necessitavam de adequação de forma farmacêutica na alta, } \\
\text { mediana }\left(I Q R^{\star}\right)\end{array}$ & 2 & [1-3] \\
\hline \multicolumn{3}{|l|}{$\%$ Medicamentos avaliados na alta e por telefone } \\
\hline Clobazam & 17 & $45,9 \%$ \\
\hline Topiramato & 11 & $29,7 \%$ \\
\hline Vigabatrina & 5 & $13,5 \%$ \\
\hline Primidona & 2 & $5,4 \%$ \\
\hline Levetiracetam & 1 & $2,7 \%$ \\
\hline Lamotrigina & 1 & $2,7 \%$ \\
\hline Total de medicamentos avaliados & 37 & $100,0 \%$ \\
\hline
\end{tabular}

*IQR: intervalo interquartílico.

Impacto da orientação farmacêutica no processo de uso de medicamentos

Conforme demonstrado no Gráfico 1 na admissão o escore médio do Medtake Test foi de
77,5 (+ 21,3). Após a orientação farmacêutica, o escore médio aumentou para 94,7 $(+9,8)$ e persistiu acima de 90 uma semana após a alta hospitalar (média $=91,8+17,4)$. 


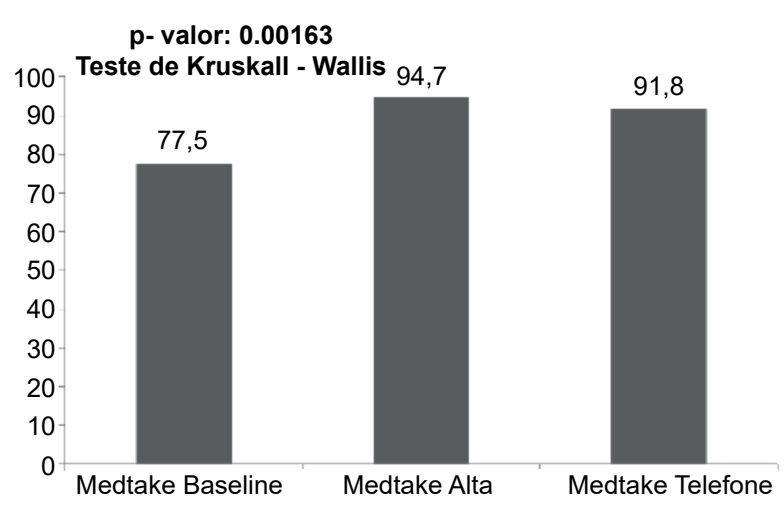

Gráfico 1: Escore médio do Medtake Test na internação, alta hospitalar e por telefone.

\section{Perfil dos erros de medicação e associações exploradas no estudo}

Os erros identificados no estudo foram classificados e quantificados conforme avaliação do instrumento Medtake Test e exemplificados na Tabela 3. Os principais tipos de erro identificados na primeira avaliação do cuidador foram os erros relacionados à administração dos medicamentos pela sonda ou gastrostomia (30\% não lavavam adequadamente a sonda ou gastrostomia na administração dos medicamentos), à utilização dos comprimidos sulcados partidos ao meio (15\%) e a administração dos medicamentos em horários inadequados (15\%). $\mathrm{Na}$ admissão a nota média, em uma escala de 0 a 10, da autoavaliação dos cuidadores sobre capacidade em administrar corretamente os medicamentos do paciente foi de $8,48(+1,64)$.

Após a orientação farmacêutica tanto no momento da alta quanto por telefone o erro mais encontrado foi a utilização dos comprimidos sulcados ao meio ( $57,10 \%$ e $44,4 \%$ dos erros respectivamente). Após a orientação farmacêutica a nota da autoavaliação foi de $9,09(+0,87)$, não houve diferença significativa entre os diferentes tempos de autoavaliações.

$\mathrm{Na}$ análise de associação entre variáveis clínicas e sócio-demográficas com o escore de Medtake, o fato de ter cuidador compartilhado esteve associado a melhores pontuações desta ferramenta $(r=0,571$ e $p=0,003$ ) no baseline, porém não no período pósalta, conforme demonstrado na Tabela 4.

Tabela 3: Descrição dos tipos de erros identificados no estudo.

\begin{tabular}{|c|c|c|c|c|c|c|c|}
\hline \multirow[b]{2}{*}{ Número de erros } & \multicolumn{2}{|c|}{ Baseline } & \multicolumn{2}{|c|}{ Após orientação } & \multicolumn{2}{|c|}{ Telefone } & \multirow[t]{2}{*}{ Experiências do estudo } \\
\hline & $\mathrm{N}=40$ & $\%$ & $\mathrm{~N}=7$ & $\%$ & $\mathrm{~N}=9$ & $\%$ & \\
\hline \multicolumn{8}{|l|}{ Erros no preparo } \\
\hline $\begin{array}{l}\text { Corta comprimido } \\
\text { sulcado ao meio }\end{array}$ & 6 & $15 \%$ & 4 & $57,10 \%$ & 4 & $44,40 \%$ & $\begin{array}{l}\text { Ao cortar o comprimido de clobazam } \\
\text { com cortador ele "esfarela". }\end{array}$ \\
\hline $\begin{array}{l}\text { Corta comprimido não } \\
\text { sulcado ao meio }\end{array}$ & 3 & $7,50 \%$ & & & & & $\begin{array}{l}\text { Ao cortar o comprimido de } \\
\text { topiramato com uma faca ocorre } \\
\text { perda do medicamento, pois o } \\
\text { comprimido esfarela. }\end{array}$ \\
\hline $\begin{array}{l}\text { Corta comprimido em } \\
\text { mais de duas partes }\end{array}$ & 2 & $5 \%$ & & & & & $\begin{array}{l}\text { Cuidador parte o comprimido } \\
\text { de clobazam em } 4 \text { partes, com } \\
\text { dificuldade de cortar o comprimido } \\
\text { em } 4 \text { partes iguais. }\end{array}$ \\
\hline Utiliza água inadequada & 1 & $2,50 \%$ & 1 & $14,30 \%$ & 1 & $11,10 \%$ & $\begin{array}{l}\text { Utilização de água da torneira para } \\
\text { dissolver os medicamentos. }\end{array}$ \\
\hline $\begin{array}{l}\text { Não homogeniza após } \\
\text { dissolução do comprimido } \\
\text { em água }\end{array}$ & 3 & $7,50 \%$ & & & & & $\begin{array}{l}\text { Deposição do medicamento no fundo } \\
\text { do frasco, com perda de dose. }\end{array}$ \\
\hline $\begin{array}{l}\text { Não mensura corretamente } \\
\text { o volume de água }\end{array}$ & & & 1 & $14,30 \%$ & & & $\begin{array}{l}\text { Cuidador não mensura o volume de } \\
\text { água que dilui o medicamento. }\end{array}$ \\
\hline $\begin{array}{l}\text { Dissolve dois } \\
\text { medicamentos juntos }\end{array}$ & 1 & $2,50 \%$ & & & & & $\begin{array}{l}\text { Diluição na mesma seringa de } \\
\text { comprimidos de risperidona e } \\
\text { clobazam. }\end{array}$ \\
\hline $\begin{array}{l}\text { Utiliza a mesma seringa } \\
\text { para administração de } \\
\text { todos os medicamentos }\end{array}$ & 3 & $7,50 \%$ & & & & & $\begin{array}{l}\text { Diluição de todos os medicamentos } \\
\text { com a mesma seringa, sem lavá-la. }\end{array}$ \\
\hline \multicolumn{8}{|l|}{ Erro na posologia } \\
\hline Horários inadequados & 6 & $15 \%$ & & & & & $\begin{array}{l}\text { Utilização domiciliar do topiramato às } \\
\text { 8h e a meia noite ao invés de } 12 / 12 \mathrm{~h} \text {. } \\
\text { Administração dos medicamentos a } \\
\text { "qualquer" hora da manhã. }\end{array}$ \\
\hline
\end{tabular}


Tabela 3: Contiuação.

\begin{tabular}{|c|c|c|c|c|c|c|c|}
\hline \multirow[b]{2}{*}{ Número de erros } & \multicolumn{2}{|c|}{ Baseline } & \multicolumn{2}{|c|}{ Após orientação } & \multicolumn{2}{|c|}{ Telefone } & \multirow[t]{2}{*}{ Experiências do estudo } \\
\hline & $\mathrm{N}=40$ & $\%$ & $\mathrm{~N}=7$ & $\%$ & $\mathrm{~N}=9$ & $\%$ & \\
\hline \multicolumn{8}{|l|}{ Erros no preparo } \\
\hline Dose/ posologia errada & 2 & $5 \%$ & & & 2 & $22,20 \%$ & $\begin{array}{l}\text { Utilização domiciliar do topiramato de } \\
12 / 12 \text { horas, porém a prescrição é de } \\
8 / 8 \text { horas. }\end{array}$ \\
\hline \multicolumn{8}{|l|}{ Erros na administração } \\
\hline $\begin{array}{l}\text { Não lava a sonda/ } \\
\text { gastrostomia } \\
\text { adequadamente } \\
\text { Indicação do } \\
\text { medicamento }\end{array}$ & 12 & $30 \%$ & 1 & $14,30 \%$ & 2 & $22,20 \%$ & $\begin{array}{l}\text { Utilização da dieta logo após a } \\
\text { administração dos medicamentos. }\end{array}$ \\
\hline Desconhece a indicação & 1 & $2,50 \%$ & & & & & $\begin{array}{l}\text { Confusão entre os medicamentos } \\
\text { ranitidina e fenitoína. }\end{array}$ \\
\hline
\end{tabular}

Tabela 4: Relação entre variáveis do paciente e escore de Medtake Test no baseline e após a alta hospitalar.

\begin{tabular}{|c|c|c|}
\hline \multicolumn{3}{|c|}{ Baseline Alta } \\
\hline Idade do cuidador & $\begin{array}{l}r=0,072 \\
p=0,733\end{array}$ & $\begin{array}{l}r=0,202 \\
p=0,367\end{array}$ \\
\hline Número de filhos & $\begin{array}{l}r=0,152 \\
p=0,467\end{array}$ & $\begin{array}{l}r=0,076 \\
p=0,736\end{array}$ \\
\hline Grau de escolaridade dos cuidadores & $\begin{array}{l}r=0,086 \\
p=0,682\end{array}$ & $\begin{array}{l}r=0,052 \\
p=0,817\end{array}$ \\
\hline Tipo de cuidado & $\begin{array}{l}r=0,571 \\
p=0,003^{*}\end{array}$ & $\begin{array}{l}r=0,198 \\
p=0,375\end{array}$ \\
\hline Tempo de uso dos medicamentos & $\begin{array}{l}r=0,049 \\
p=0,815\end{array}$ & $\begin{array}{l}r=0,028 \\
p=0,901\end{array}$ \\
\hline Número de medicamentos em uso & $\begin{array}{l}r=0,339 \\
p=0,097\end{array}$ & $\begin{array}{l}r=0,387 \\
p=0,075\end{array}$ \\
\hline $\begin{array}{l}\text { Número de medicamentos em uso que necessitavam } \\
\text { adequacaão de forma farmacêutica. }\end{array}$ & $\begin{array}{l}r=0,106 \\
p=0,613\end{array}$ & $\begin{array}{l}r=0,289 \\
p=0,192\end{array}$ \\
\hline
\end{tabular}

Correlações realizadas por meio de teste de Spearman, com significância estatística se $p<0,05\left(^{*}\right)$.

Observações: o grau de escolaridade foi categorizado em (1) nível ensino fundamental ou inferior; (2) ensino médio; (3) ensino superior. O tipo de cuidado foi categorizado em (1) exclusivo e (2) compartilhado.

\section{DISCUSSÃO}

Esse é o primeiro estudo avaliando o impacto da orientação do farmacêutico na melhora do processo de uso de anticonvulsivantes em pacientes com epilepsia refratária, visto a prescrição recorrente de formas farmacêuticas inadequadas para pacientes pediátricos no momento da alta hospitalar. Ressalta-se a melhora do escore médio do Medtake Test dos cuidadores, a identificação e prevenção de erros relacionados ao uso do tratamento e a possibilidade de uso dos achados deste estudo de viabilidade em estudos de intervenção futuros, como definição da magnitude da melhora do escore de Medtake e relevância clínica dos erros de medicamentos encontrados.

A melhora absoluta atribuível à orientação farmacêutica de alta hospitalar neste estudo exploratório foi de $15 \%(p=0,02)$ no escore Medtake Test, onde 19 dos 25 cuidadores avaliados no estudo possuíam ao menos 1 erro relacionado ao processo de uso dos anticonvulsivantes, totalizando 40 erros.
Destes 40 erros destacam-se a administração inadequada dos medicamentos pela sonda e gastrostomia, utilização de comprimidos sulcados partidos ao meio e administração dos medicamentos em horários inadequados, principalmente antes da orientação farmacêutica.

A administração inadequada dos medicamentos pela sonda ou gastrostomia, tanto pela lavagem inadequada desta após a administração dos medicamentos ou da dieta enteral, quanto pela administração concomitante de medicamentos pode contribuir para efeitos adversos mensuráveis. Tais desfechos vão desde a obstrução de sondas até a alteração de efeito do medicamento, levando a redução da eficácia ou toxicidade dos medicamentos ${ }^{16}$. Pesquisas sugerem que menos de $50 \%$ das sondas são lavadas adequadamente entre a administração de dois medicamentos e este processo reduz a incidência de obstrução da sonda ${ }^{17,18}$. Após a alta apenas dois medicamentos continuaram sendo administrados erroneamente, isto demonstra a 
importância do reforço desta prática no momento da orientação de alta. Um estudo demonstrou que as orientações para pacientes dadas por enfermeiros e farmacêuticos acerca do uso dos medicamentos pela sonda foram eficazes para uma melhoria na administração e redução significativa do número de obstruções ${ }^{19}$.

Muitos pais desconhecem a importância da administração dos anticonvulsivantes em horários adequados conforme a prescrição médica e a manutenção diária dos mesmos horários para garantir a ação efetiva dos medicamentos no controle das crises convulsivas e melhorar a adesão ao tratamento. A organização destes conforme a rotina da família pode auxiliar a redução de omissão de doses, que é significativamente atribuída à ocorrência de crises convulsivas ${ }^{20}$.

A divisão dos comprimidos foi um dos erros frequentemente identificados no estudo, visto que é rotina usual entre pediatras esta orientação como forma de adequação da dose, dada a indisponibilidade de formas farmacêuticas líquidas. Sabe-se que a divisão do comprimido de anticonvulsivantes leva a uma falta de uniformidade de peso ou de conteúdo do princípio ativo ao fracionar o comprimido ${ }^{21}$. Uma revisão recente realizada por Richey et al. em 2017 demonstrou que há uma escassez de evidências para apoiar a manipulação de drogas em crianças, sendo necessário avaliar o impacto da manipulação na precisão da dosagem e a necessidade da própria indústria farmacêutica incluir nos programas de desenvolvimento de medicamentos o efeito da manipulação dos comprimidos ${ }^{22}$.

Nestes pacientes que utilizam terapia combinada com diversos medicamentos que necessitam de manipulação, as variações de doses podem influenciar fortemente o sucesso no controle das crises convulsivas em casos de administração de subdoses como também contribuir para a ocorrência de efeitos adversos relacionados à sobredose dos anticonvulsivantes, tais como: hipotensão, bradicardia, sonolência e sedação. Sabe-se que os efeitos adversos são fatores predisponentes para a não adesão ao tratamento ${ }^{1,4,23}$. Ambas as situações podem resultar em necessidade de ajustes na terapia, inclusão ou exclusão de medicamentos, entretanto tais desfechos estão muitas vezes relacionados exclusivamente a má utilização dos medicamentos. Em visitas domiciliares a famílias de pacientes pediátricos com epilepsia, Walsh et al. em 2011 identificaram que os erros envolvendo medicamentos mais comuns foram os de administração (15\%) geralmente associados a trocas de dose dos anticonvulsivantes ${ }^{24}$.

Optamos por não definir um corte do escore Medtake por não haver definição ou estudo que demonstre uma associação entre escores de Medtake e desfechos clínicos em pacientes pediátricos com epilepsia refratária. Adicionalmente, buscamos avaliar variáveis sociodemográficas e clínicas dos pacientes e cuidadores e sua relação com escore de Medtake. Gomes et al, por exemplo encontraram idade $>70$ anos, analfabetismo e menor tempo de uso dos medicamentos como fatores relacionados a piores escores de Medtake $(<80 \%)^{25}$. Apesar de tais relatos na literatura, a única associação encontrada foi o cuidado compartilhado no momento baseline, entretanto após a alta hospitalar não observamos tal associação ${ }^{2,14}$. Atribuímos que a capacidade em seguir corretamente a terapia é multifatorial e envolve muitos fatores individuais que não puderam ser mensurados e comparados. Estes fatores podem variar, por exemplo, com o modo de organização familiar no cuidado do paciente e até mesmo pela da comunicação do profissional de saúde com o cuidador.

Estudos prévios sugerem que a orientação farmacêutica em pediatria aumenta a apropriação, o conhecimento e a satisfação dos pais em relação ao tratamento ${ }^{26,27}$. A grande limitação destes estudos, assim como nos estudos com educação farmacêutica para população adulta, é a falta de descrição da ferramenta de educação utilizada. Pela nossa experiência, na alta hospitalar as famílias recebem diversas orientações ao mesmo tempo por diferentes profissionais, levando a uma sobrecarga de informações que pode potencializar o risco para ocorrência de erros envolvendo medicamentos no retorno para casa. Em 2016, Schuh et al. ${ }^{27}$ relataram que mais de $80 \%$ dos pais sentiam-se preparados para ir para casa e relatavam ter entendido completamente as orientações fornecidas sobre os medicamentos, e mesmo assim $20 \%$ apresentaram problemas após a alta pelos quais não esperavam. ${ }^{27} \mathrm{~A}$ orientação continuada pelos profissionais da saúde é fundamental, ela pode e deve ser realizada da admissão à alta hospitalar, uma vez que todos os momentos de contato com a família são oportunidades para reforçar orientações e sanar dúvidas, sempre com uma linguagem adequada e de fácil compreensão, utilizando a técnica "teach back" para maior aproximação do profissional com o cuidador, tornando a educação mais efetiva. ${ }^{14,28}$

Este estudo vem a contribuir para a literatura com a magnitude do efeito atribuível á orientação do farmacêutico após orientação sobre o processo de uso de medicamentos em cuidadores, ainda que não se saiba o impacto disto na taxa de reinternação ou no número de convulsões, a orientação é feita de modo a aumentar a segurança no uso dos medicamentos no domicílio. Diante da inexistência de estudos sobre o tema proposto, qualquer calculo amostral seria considerado teórico. Com o presente estudo, é possível sugerir que é esperada uma melhora de $15 \%$ do escore de Medtake após a orientação de um farmacêutico clínico, sendo este um parâmetro essencial para cálculo amostral. Não obstante, pesquisas de intervenção ou melhoria 
do uso de anticonvulsivantes poderão aprimorar o conhecimento e contribuir para melhorar os erros encontrados neste estudo.

Por ser um estudo de viabilidade, o mesmo não possui o objetivo de testar hipóteses, mas de explorar dados voltados à pesquisa sobre o uso correto de anticonvulsivantes. É importante frisar que o Medtake Test foi desenvolvido para avaliar a capacidade de adultos seguirem corretamente a terapia e sua validação para a língua portuguesa não foi realizada, entretanto devido à limitação de literatura envolvendo o tema e esta população, optamos por utilizá-lo para maior aproximação da realidade. Além disso, não foi avaliada a melhora clínica dos pacientes, sendo essas as oportunidades para estudos futuros. Salientamos como limitação do instrumento que todos os parâmetros avaliados são pontuados igualmente, entretanto estes podem apresentar relevâncias clínicas diferentes, por este motivo os erros encontrados no estudo não reportam a total incapacidade de administrar os medicamentos.
Conclui-se que a orientação de farmacêuticos clínicos a cuidadores de pacientes com epilepsia refratária possui o potencial de aumentar o uso correto de medicamentos mensurados pelo Medtake Test em 15\%, considerando que todos os erros são evitáveis, a orientação farmacêutica pode contribuir para a segurança do uso dos medicamentos após a alta e com isso trazer um potencial benefício clínico na alta hospitalar dos pacientes pediátricos.

\section{Financiamento}

Os recursos necessários para realização do trabalho foram custeados pelos pesquisadores e pelo Hospital de Clínicas de Porto Alegre.

\section{Conflitos de Interesse}

Não há conflitos de interesse específicos para esse estudo.

\section{Referências}

1. Lee YK, Ah YM, Choi YJ, Cho YS, Kim KJ, Lee JY. Antiepileptic drug adherence and persistence in children with epilepsy attending a large tertiary care children's hospital. Epileptic Disord. 2016;1(4):408-17.

2. Modi AC, Rausch JR, Glauser TA. Early pediatric antiepileptic drug nonadherence is related to lower long-term seizure freedom. Neurology. 2014;82:671-3

3. LaRoche SM, Helmers SL. The New Antiepileptic Drugs. JAMA. 2004;291(5):605-14.

4. Sabaté E. Epilepsy. In: Sabaté, E. Adherence to long-term therapies: evidence for action [Internet]. Geneva: World Health Organization; 2003 [citado 25 out 2018]. p. 87-93. Disponível em: http://www.who.int/chp/ knowledge/publications/adherence full_report.pdf?ua=1

5. Farley TM, Shelsky C, Powell S, Farris KB, Carter BL. Effect of clinical pharmacist intervention on medication discrepancies following hospital discharge. Int J Clin Pharm. 2014;36(2):430-7.

6. Sánchez UA, Gallardo LS, Pons LN, Murgadella SA, Campins BL, Merino MR. Pharmaceutical intervention upon hospital discharge to strengthen understanding and adherence to pharmacological treatment. Farm Hosp. 2012;36(3):118-23.
7. Sarangarm $P$, London MS, Snowden SS, Dilworth TJ, Koselke LR, Sanchez CO, et al. Impact of pharmacist discharge medication therapy counseling and disease state education: Pharmacist Assisting at Routine Medical Discharge (project PhARMD). Am J Med Qual. 2013; 28(4):292-300.

8. Chen C, Lee DS, Hie SL. The impact of pharmacist's counseling on pediatric patients' caregiver's knowledge on epilepsy and its treatment in a tertiary hospital. Int J Clin Pharm. 2013;35(5):829-34.

9. Auger KA, Kenyon CC, Feudtner C, Davis MM. Pediatric Hospital Discharge Interventions to Reduce Subsequent Utilization: A Systematic Review. J Hosp Med. 2014;9(4):251-60.

10. Leathers L, Brittain KL, Crowley K. Effect of a Pediatric Prescription Medication Discharge Program on Reducing Hospital Readmission Rates. J Pediatr Pharmacol Ther. 2017;22(2):94-101.

11. Wu S, Tyler A, Logsdon T, Holmes NM, Balkian A, Brittan M, et al. A Quality Improvement Collaborative to Improve the Discharge Process for Hospitalized Children. Pediatrics. 2016;138(2):e20143604.

12. Mallory LA, Diminick NP, Bourque JP, Bryden MR, Miller JL, Nystrom NM, et al. Pediatric Patient-Centered
Transitions From Hospital to Home: Improving the Discharge Medication Process. Hospital Pediatr. 2017;7(12):723-30.

13. Okumura LM, Rotta I, Correr CJ. Assessment of pharmacist-led patient counseling in randomized controlled trials: a systematic review. Int J Clin Pharm. 2014;36(5):882-91.

14. Raehl CL, Bond CA, Woods T, Patry RA, Sleeper RB. Individualized Drug Use Assessment in the Elderly. Pharmacotherapy. 2002;22(10): 1239-1248.

15. White, R. Choice of medication formulation. In: White R, Bradnam V. Handbook of Drugs Administration via Enteral Feeding Tubes. 3. ed. London: Pharmaceutical Press; 2015. p. 25-37.

16. Bankhead R, Boullata J, Brantley S, Corkins M, Guenter P, Krenitsky $J$, et al. Enteral nutrition practice recommendations. J Parenter Enteral Nutr. 2009;33:122-67.

17. Sohrevardi SM, Jarahzadeh $M H$, Mirzaei E, Mirjalili M, Tafti AD, Heydari B. Medication errors in patients with enteral feeding tubes in the intensive care unit. J Res Pharm Pract. 2017;6(2):100-5.

18. Scanlan M, Frisch S. Nasoduodenal feeding tubes: prevention of occlusion. J Neurosci Nurs.1992;24:256-9. 
19. van den Bemt PM, Cusell MB, Overbeeke PW, Trommelen M, van Dooren D, Ophorst WR, et al. Quality improvement of oral medication administration in patients with enteral feeding tubes. Qual Saf Health Care. 2006;15(1):44-7.

20. Cramer JA, Glassman M, Rienzi $V$. The relationship between poor medication compliance and seizures. Epilepsy Behav. 2002;3(4):338-42.

21. Nidanapu RP, Rajan S, Mahadevan S, Gitanjali B. Tablet splitting of antiepileptic drugs in pediatric epilepsy: potential effect on plasma drug concentrations. Pediatr Drugs. 2016;18:451-63.

22. Richey RH, Hughes C, Craig JV, Shah UU, Ford JL, Barker CE, et al. A systematic review of the use of dosage form manipulation to obtain required doses to inform use of manipulation in pediatric practice (Journal article). Int J Pharm. 2017;518:155-66.

23. Patsalos PN, Berry DJ, Bourgeois BFD, Cloyd JC, Glauser TA, Johannessen SI, et al. Antiepileptic drugs - best practice guidelines for therapeutic drug monitoring: a position paper by the subcommission on therapeutic drug monitoring. ILAE Commission on Therapeutic Strategies. Epilepsia. 2008;49:1239-76.

24. Walsh KE, Mazor KM, Stille CJ, Torres I, Wagner JL, Moretti J, et al. Medication errors in the homes of children with chronic conditions. Arch Dis Child. 2011;96:581-6.

25. Gomes DC, Paiva LC, Farhat FCLG, Pedro AO, Neto AMP. Ability to follow anti-reabsorptive drug treatment in postmenopausal women with reduced bone mass. Menopause. 2011;18(5):531-6.

26. Angoulvant $F$, Rouault A, ProtLabarthe S, Boizeau P, Skurnik D, Morin L, et al. Randomized controlled trial of parent therapeutic education on antibiotics to improve parent satisfaction and attitudes in a pediatric emergency department. PLoS One. 2013;8(9):e75590.

27. Schuh M, Schendel S, Islam S, Klassen K, Morrison L, Rankin KN, et al. Parent readiness for discharge from a tertiary care pediatric cardiology unit. J Spec Pediatr Nurs. 2016;21(3):139-46.

28. Cua MY, Kripalani S. Medication Use in the Transition from Hospital to Home. Ann Acad Med Singapore. 2008;37(2):136- 41.

Recebido 13 jan, 2020 Aceito: 9 fev, 2021 